Major Article

\title{
Variability in practice patterns regarding protective isolation measures after heart transplantation: A secondary analysis of the international BRIGHT study
}

\author{
Ellen Alaerts MNS a , Carolien Dreesen MNS a , Kris Denhaerynck PhD ${ }^{\mathrm{b}}$, Sabine Gryp MNS ${ }^{\mathrm{c}}$, \\ Johan Van Cleemput PhD, MD ${ }^{\mathrm{c}}$, Annette Schuermans PhD, MD, MSc ${ }^{\mathrm{d}}$, Cynthia L. Russell PhD, RN ${ }^{\mathrm{e}}$, \\ Fabienne Dobbels PhD ${ }^{\mathrm{a}, \mathrm{b}}$, Sabina De Geest PhD, RN ${ }^{\mathrm{a}, \mathrm{b}, *}$, for the BRIGHT study team \\ ${ }^{a}$ Academic Center for Nursing and Midwifery, Department of Public Health and Primary Care, Faculty of Medicine, KU Leuven, Leuven, Belgium \\ ${ }^{\mathrm{b}}$ Institute of Nursing Science, Department Public Health, Faculty of Medicine, University of Basel, Basel, Switzerland \\ ${ }^{\mathrm{c}}$ Heart Transplant Program, University Hospitals of Leuven E' Department Cardiovascular Sciences, KU Leuven, Leuven, Belgium \\ ${ }^{\mathrm{d}}$ Department of Hospital Hygiene and Epidemiology, University Hospitals of Leuven, Leuven, Belgium \\ e School of Nursing and Health Studies, University of Missouri-Kansas City, Kansas City, MO, USA
}

Key Words:

Infection control

International comparison

Standard precaution measures

Immunosuppression

Disinfection

Private room

\begin{abstract}
Background: Infection control is a cornerstone of post-heart transplantation (HTx) in-hospital management when immunosuppression is highest. The use of protective isolation persists despite its questionable effectiveness. We describe and compare practice patterns internationally and assessed correlates of protective isolation.

Methods: Using the BRIGHT-study data, a cross-sectional intercontinental study, we assessed 12 protective isolation measures in 4 continents, 11 countries, and 36 HTx centers. Data were summarized descriptively, as appropriate. Comparisons between countries and continents and association testing between center characteristics and number of isolation measures used were also explored by general linear modeling.

Results: A total of $89 \%$ (32/36) of HTx centers used protective isolation measures with an average of 4.5 protective isolation measures per center (SD, 2.6; range 1-10). Most often applied were disinfecting high-touch surfaces $(n=27 / 34 ; 79.4 \%)$, use of private room $(n=27 / 36 ; 75.0 \%)$, and changing linen daily $(n=25 / 36$; $69.4 \%)$. Least applied were wearing a cap $(n=6 / 35 ; 17.1 \%)$ and high-efficiency particulate air filtration $(\mathrm{N}=5 / 32 ; 15.6 \%)$. Larger centers and those with dedicated beds for HTx applied more isolation measures.
\end{abstract}

\footnotetext{
*Address correspondence to Sabina De Geest, PhD, RN, Institute of Nursing Science, Department Public Health, University of Basel, Bernoullistrasse 28, CH-4056 Basel Switzerland.

E-mail address: sabina.degeest@unibas.ch (S. De Geest).

The BRIGHT Study team consists of: Marisa G. Crespo-Leiro (Complexo Hospitalario Universitario A Coruña (CHUAC), CIBERCV, INIBIC, Universidade da Coruña (UDC), La Coruña, Spain); Sandra Cupples (US Department of Veterans Affairs, Veteran Health Administration, Washington DC, USA); Paolo De Simone (Azienda Ospedaliero-Universitaria Pisana, Ospedale Cisanello, Pisa, Italy); Albert Groenewoud (Astellas Pharma Europe Ltd, UK); Christiane Kugler (Hannover Medical School, Hannover Germany); Linda Ohler (George Washington University, Washington DC, USA); Johan Van Cleemput (University Hospitals Leuven, Leuven, Belgium); Alain Jean Poncelet (Cliniques Universitaires Saint-Luc, Brussels, Belgium); Laurent Sebbag (Hôpital Louis Pradel, Lyon, France); Magali Michel (Hôpital Nord Laennec, Nantes, France); Andrée Bernard (Hôpital Universitaire Pitié-Salpêtrière, Paris, France); Andreas Doesch (University Hospital Heidelberg, Heidelberg, Germany); Ugolino Livi (University Hospita Udine, Udine, Italy); Luciano Potena (University of Bologna, Bologna, Italy); Vicens Brossa-Loidi (Hospital de Sant Pau, Barcelona, Spain); Javier Segovia-Cubero (Hospita Puerta de Hierro, Madrid, Spain); Luis Almenar-Bonet (Hospital Universitari i Politècnic La Fe de Valencia, Valencia, Spain); Carmen Segura Saint-Gerons (Hospital Univeritario Reina Sofia, Córdoba, Spain); Paul Mohacsi (University Hospital of Bern, Bern Switzerland); Eva Horvath (University Hospital Zurich, Zurich, Switzerland); Cheryl Riotto (Papworth Hospital, Cambridge, UK); Gareth Parry (Freeman Hospital,
}

Newcastle, UK); Ashi Firouzi (Royal Brompton \& Harefield NHS Foundation Trust, London, UK); Stella Kozuszko (Toronto General Hospital, Toronto, Canada); Haissam Haddad (University of Ottawa Heart Institute, Ottawa, ON, Canada); Annemarie Kaan (St Paul's Hospital, Vancouver, BC, Canada); Grant Fisher (London Health Sciences Centre, London, ON, Canada); Tara Miller (Duke University Hospital, Durham, NC, USA); Maureen Flattery (Virginia Commonwealth University Health System, Richmond, VA, USA); Kristin Ludrosky/Nancy Albert (Cleveland Clinic, OH, USA); Bernice Coleman (Cedars-Sinai Medical Center, Los Angeles, CA, USA); Jacqueline Trammell \& Flavio Epstein (Kaiser Permanente Santa Clara Medical Center, Santa Clara, CA, USA); Katherine St. Clair, Andrew Kao (St. Luke's Hospital, Kansas City, MO, USA); Maria Molina (Hospital of the University of Pennsylvania, Philadelphia, PA, USA); Karyn Ryan Canales (Ochsner Medical Center, New Orleans, LA, USA); Samira Scalso de Almeida (Hospital Israelita Albert Einstein, São Paulo \& Hospital Municipal Vila Santa Catarina - Ministerio da Saude PROAD/-SUS, São Paulo, Brazil); Bartira de Aguiar Roza, Paulista School of Nursing, Federal University of Sao Paulo, São Paolo, Brazil; Andrea Cotait Ayoub (Instituto Dante Pazzanese de Cardiologia, São Paulo, Brazil); Fernanda Barone (Instituto do Coração da Universidade de São Paulo, São Paulo Brazil); Michelle Harkess (St. Vincent's Hospital, Sydney, Australia); Joanne Maddicks-Law (The Prince Charles Hospital, Brisbane, Australia).

Conflicts of interest: None to report.

Author contributions: E.A. and C.D. are joint first authors. F.D. and S.D.G. are joint last authors. 
Conclusions: Protective isolation measures are still widely applied within heart transplant centers across the world persists notwithstanding its doubtful effectiveness. Future clinical guidelines for heart transplant management should include a statement of the need for strict adherence to standard infection prevention measures.

(c) 2020 Published by Elsevier Inc. on behalf of Association for Professionals in Infection Control and Epidemiology, Inc.

Heart transplant recipients receive high doses of immunosuppressive medications to prevent rejection, making them susceptible for infection. Infections remain the main cause of morbidity and mortality in the first year post-heart transplantation (HTx) ${ }^{1}$ According to the Registry of the International Society for Heart and Lung Transplantation, $13.9 \%$ of deaths occurring within the first month HTx are attributable to infections. ${ }^{2}$ Most of the infections arise immediately post-transplant are hospital acquired and are of bacterial etiology, followed by opportunistic infections (eg, reactivations of herpes simplex). ${ }^{3}$ Beyond the first month, opportunistic and viral infections are the most common, and account for about one-third of all deaths occurring between 1 and 12 months post-transplant. $^{2}$

To protect these immunocompromised patients, heart transplant centers typically apply infection prevention measures during the immediate inpatient period. Prevention measures can range from standard precautions to strict protective isolation. ${ }^{4-6}$ Standard precautions refer to hand hygiene, respiratory hygiene, and wearing personal protective equipment at time of exposure to body substances or to contaminated surfaces. Protective isolation measures might include private room; changing linen daily; wearing gloves, gown, cap, and mask when entering the room; disinfection of material and high-touch surfaces; uni-dose packaging; or food safety measures, as well as the installation of high-efficiency (> 90\%) particulate air filter (HEPA) and laminar air flow.

Whereas the use of standard precautions is undebated, the effectiveness of protective isolation measures during the in-hospital phase HTx remains unclear. To our knowledge, only 3 studies, from the early days of transplantation and of suboptimal methodological quality, did not show superior efficacy of protective isolation in preventing infections or improving survival compared with standard precaution measures. ${ }^{5,7,8}$ Guidelines for HTx management do not specify recommendations for protective isolation measures. ${ }^{6}$ Therefore, it remains unclear how many and which heart transplant centers continue to use protective isolation measures.

Given the lack of international comparative data regarding the practice patterns of use of protective isolation measures in heart transplant programs, we aimed to: (1) describe the use of various protective isolation measures in HTX centers internationally; (2) compare the use of protective measures between countries and continents; and ( 3 ) assess whether center characteristics correlate with the number of protective isolation measures used.

\section{METHODS}

\section{Design and setting}

This study is a secondary analysis of the BRIGHT (Building Research Initiative Group: chronic illness management and adherence in Transplantation) study that used a cross-sectional design and included a convenience sample of 36 HTx centers from 11 countries in 4 continents. Therefore, only heart transplant patients were included in our study. The methodological details of the BRIGHT study have been previously reported. ${ }^{9}$ We summarize the relevant methodological information for the present study below.

\section{Sample}

The BRIGHT study used a multi-staged sampling approach: Per selected country (convenience sample), a minimum of 2 HTx centers were included using following inclusion criteria: $(1) \geq 50$ heart transplant procedures performed during the last 12-60 months before data collection was started; (2) located in Australia, Europe, North or South America; and (3) the responsible administrator and transplant director provided written approval for participation in the BRIGHT study.

\section{Variables and measurement}

Practice patterns in view of standard precautions ( 1 item, ie, hand hygiene) and protective isolation measures (12 items) were assessed using an investigator-developed instrument based on the literature and clinical experience of the BRIGHT study team. The instrument used a dichotomous answering option (yes/no) (Table 2). A total score was also calculated by aggregating the number of protective isolation measures applied per center. Data per country and continent were presented as mean scores, by averaging the within-country center scores, and within-continent center scores, respectively.

The following HTx center characteristics were assessed as potential correlates of the number of protective isolation measures used: transplant director indicated number of heart transplants for the 5 years preceding data collection, location of HTx center (urban/ suburban/rural), type of HTx center (university teaching hospital/ regional or community hospital/other), as well as the presence of dedicated beds for HTx patients on a unit (yes/no). The size of the HTx program was subsequently categorized by the researchers based on the criteria of the International Society for Heart and Lung Transplant criteria ${ }^{10}$ into small HTx centers (ie, having performed 50-74 HTx procedures over the past 5 years), medium (75-100 procedures), and large HTx centers (ie, having performed more than 100 heart transplantations over the past 5 years).

\section{Data analysis}

Descriptive statistics were used as appropriate based on the measurement level and distribution of variables (ie, frequencies, proportions, means with SDs, and range). Data were measured at center level, and subsequently aggregated at country and continent level.

Differences between countries and continents, with regard to the number of isolation measures, and its associations with center characteristics, were explored by means of general linear modeling. The significance level was set at a $P$ value of $<.05$. Data were analyzed using the Statistical Package for the Social Sciences (IBM Corporation, Armonk, NY).

\section{RESULTS}

\section{Sample characteristics}

A total of 36 HTx centers participated in this study. Centers were distributed over following countries: Belgium $(\mathrm{N}=2)$, France $(\mathrm{N}=3)$, Germany $(\mathrm{N}=2)$, Italy $(\mathrm{N}=2)$, Spain $(\mathrm{N}=5)$, Switzerland $(\mathrm{N}=2)$, 
Table 1

Characteristics of the participating centers $(\mathrm{N}=36)$

\begin{tabular}{llrr}
\hline Center characteristic & & N & \multicolumn{2}{c}{$\%$} \\
\hline Continent & Europe (n, \%) & 19 & $52.8 \%$ \\
& North America (n, \%) & 12 & $33.3 \%$ \\
& South America (n, \%) & 3 & $8.3 \%$ \\
Size* & Australia (n, \%) & 2 & $5.6 \%$ \\
& Small (n, \%) & 5 & $13.9 \%$ \\
Location & Medium (n, \%) & 8 & $22.2 \%$ \\
& Large (n, \%) & 23 & $63.9 \%$ \\
Type & Urban (n, \%) & 32 & $88.9 \%$ \\
& Suburban (n, \%) & 3 & $8.3 \%$ \\
& Rural (n, \%) & 1 & $2.8 \%$ \\
Dedicated beds for HTx patients & University (n, \%) & $83.3 \%$ \\
& Regional/Community (n, \%) & 4 & $11.1 \%$ \\
& Other (n, \%) & 2 & $5.6 \%$ \\
& & 22 & $61.1 \%$ \\
\hline
\end{tabular}

$H T x$, post-heart transplantation.

*Small, 50-74 HTx/5 years; medium. 75-100 HTx/5 years; and large, > $100 \mathrm{HTx} / 5$ years. ${ }^{10}$

United Kingdom $(\mathrm{N}=3)$, United States $(\mathrm{N}=8)$, Canada $(\mathrm{N}=4)$, Australia $(\mathrm{N}=2)$, and Brazil $(\mathrm{N}=3)$. Table 1 summarizes the characteristics of the 36 HTx centers. The majority of centers were located in Europe $(\mathrm{N}=19 ; 52.8 \%)$ or North America $(\mathrm{N}=12 ; 33.3 \%)$, were large centers $(n=23 ; 63.9 \%)$, university teaching hospitals $(n=30 ; 83.3 \%)$, and were located in urban areas $(n=32 ; 88.9 \%)$. Twenty-two centers (61.1\%) had a hospital ward with dedicated beds for post-HTx patients.

\section{Practice patterns in view of standard precautions}

All HTx centers reported using hand hygiene as a standard precaution (100\%). However, 4 centers (11.1\%) did not use protective isolation measures at all (ie, 2 centers in North America and 2 centers in Europe).

\section{Practice patterns in view of protective isolation measures}

Figure 1 shows the practice patterns in view of protective isolation measures. The most frequently applied measures were disinfecting high-touch surfaces $(\mathrm{n}=27 / 34 ; 79.4 \%)$, use of private room $(n=27 / 36 ; 75.0 \%)$, and changing linen daily $(n=25 / 36 ; 69.4 \%)$. The least applied measures were wearing a cap $(n=6 / 35 ; 17.1 \%)$ and HEPA filtration ( $\mathrm{N}=5 / 32 ; 15.6 \%)$.

\section{Variability between countries and continents in protective isolation measures used}

Table 2 presents the use of specific protective isolation measures as well as the average number of measures used per country and continent. The average number of protective isolation measures differed between countries $(P=.03)$, with the highest for Italy (mean $=8.0$; $\mathrm{SD}=1.4$ ) and France (mean $=6.7 ; \mathrm{SD}=3.2$ ), and the lowest for Canada ( mean $=1.5 ; \mathrm{SD}=1.9$ ) and Germany (mean $=2.0 ; \mathrm{SD}=2.8$ ). Looking at continent level, the mean number of protective isolation measures was the highest for Australia (mean =5.5; $\mathrm{SD}=2.1$ ), followed by Europe (mean $=5.2 ; \mathrm{SD}=2.9$ ), South America (mean = 3.7; $\mathrm{SD}=2.5$ ),

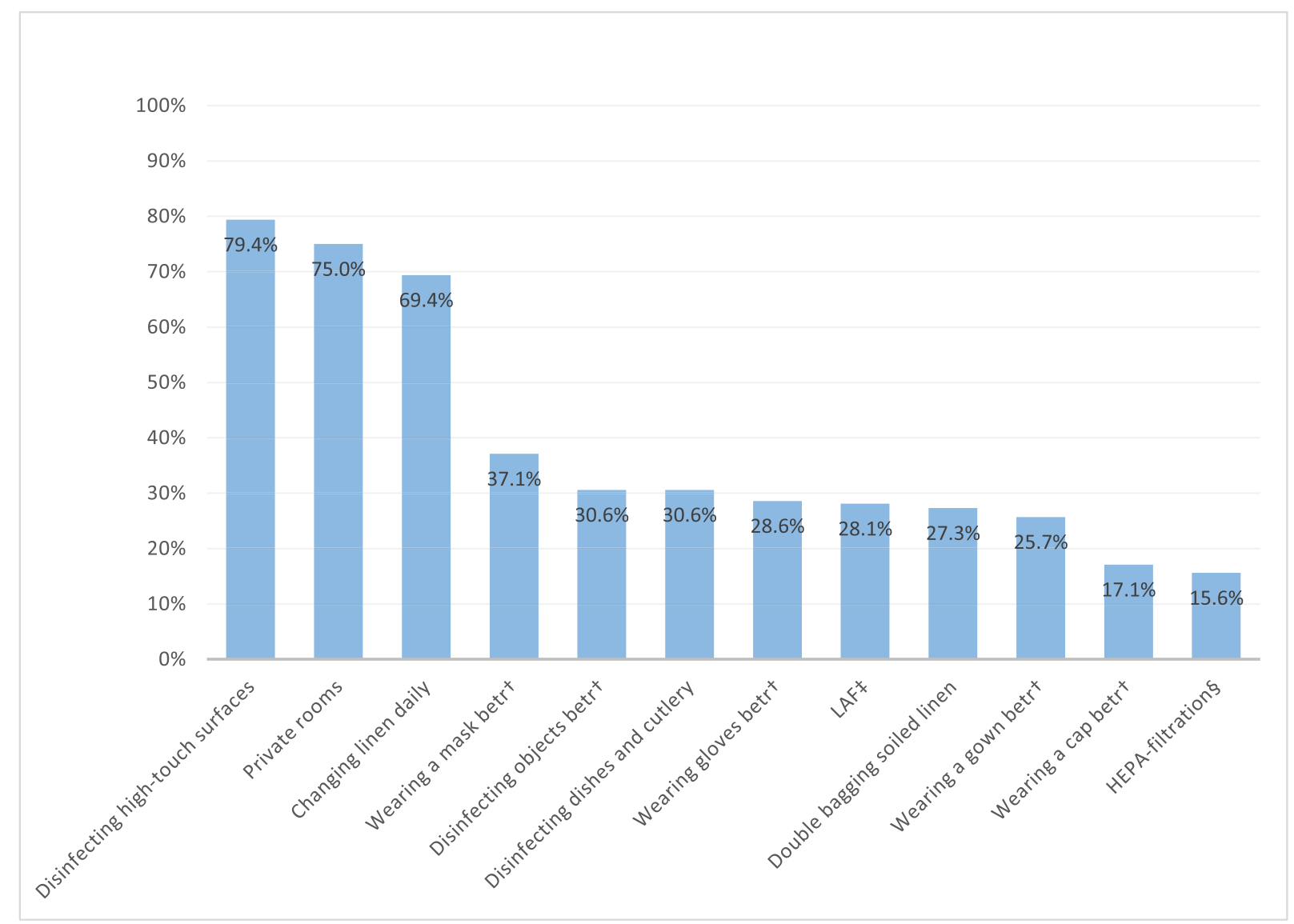

Fig 1. Prevalence of protective isolation measures at center level $(n=36)$.

Before entering the room $(\dagger)$, Laminar airflow $(\ddagger)$, and HEPA-filtration, high-efficiency $(>90 \%)$ particulate air filter $(\S)$. 
and North America (mean $=3.5 ; \mathrm{SD}=2.1$ ). These differences, however, were not significantly different $(P=.32)$.

\section{Correlates of protective isolation measures used}

Of the center characteristics explored, only the size of the HTx center was positively associated with the average number of isolation measures applied $(P=.0017)$ (Table 3$)$. The number of measures increases by an average 1.8 per 1-unit increase in size rank (eg, small HTx centers use on average 1.8 protective isolation measures less than medium-size centers).

\section{DISCUSSION}

To our knowledge, this study is the first to report international practice patterns regarding protective isolation measures during post-operative in-hospital recovery after HTx. Using data from the international BRIGHT study, including 36 HTx centers in 11 countries and 4 continents, we showed variability in clinical practice patterns regarding the use of protective isolation measures. Previous research on practice patterns in infection prevention and control in transplant management focused on donor evaluation, cytomegalovirus management, and prevention measures with dental procedures. ${ }^{11-13}$ Our work adds another important piece of evidence by zooming in on the differences in practice patterns in view of practice patterns regarding protective isolation measures between heart transplant centers, countries, and continents.

More specifically, despite the lack of evidence on the effectiveness of protective isolation measures for preventing infections in heart transplantation, ${ }^{5-8}$ their use is still widespread among heart transplant centers. More specifically, only $11 \%$ did not use protective isolation measures, and an average of 4.5 measures per center were used. Variation between countries and continents was observed, yet did not reach statistical significance. Most often applied were disinfecting high-touch surfaces, use of private rooms, and changing linen daily. The least applied were wearing a cap and HEPA filtration. Private rooms increase the physical barriers for transmission and contamination among patients, yet strict adherence to standard precautions remains unconditionally important. However, the need to disinfect high-touch surfaces beyond the standard cleaning procedures and daily changing of linen in the HTx population can be questioned. Further research is needed to investigate whether these time-consuming and costly measures help to reduce the number of infections immediately post-transplant. Other protective isolation measures could be necessary depending on the circumstances of the transplant unit and center. HEPA-filtration, for instance, can be used when the location of the transplant unit within the hospital is suboptimal (eg, close to units with a high infection rate, or where there is a frequent passage of visitors and caregivers) or during construction work. ${ }^{1}$ Unfortunately, we do not have additional information on the reasons why centers may or may not use certain protective isolation measures. Surprisingly, we observed a positive association between center size and the number of protective isolation measures used. Unfortunately, we do not have data to support these findings and can only speculate about these findings.

Future clinical guidelines for heart transplant management could include a statement of the need for strict adherence to standard infection prevention measures. The use of protective isolation measures can currently not be supported by evidence. ${ }^{5-8}$ Transplant programs could also consider de-implementing protective isolation measures during the in-hospital post-operative care to save costs. 
Table 3

Association between HTx center characteristics and mean number of protective isolation measures used per center

\begin{tabular}{|c|c|c|c|c|}
\hline Characteristic of the 36 HTx centers & & $\begin{array}{l}\text { Mean } \pm \text { SD no. of } \\
\text { measures per center }\end{array}$ & $\begin{array}{l}\text { ß } \\
\text { (95\% confidence interval) }\end{array}$ & $P$ value \\
\hline \multirow[t]{3}{*}{ Size } & Small $(\mathrm{n}=5)$ & $2.2 \pm 1.6$ & $1.79(0.72-2.86)$ & .0017 \\
\hline & Medium $(n=8)$ & $3.1 \pm 1.5$ & - & \\
\hline & Large $(n=23)$ & $5.5 \pm 2.6$ & - & \\
\hline \multirow[t]{2}{*}{ Presence of dedicated beds for HTx patients } & Yes $(n=22)$ & $4.1 \pm 2.8$ & $1.05(-0.75-2.86)$ & .24 \\
\hline & No $(n=14)$ & $5.1 \pm 2.3$ & - & \\
\hline \multirow[t]{3}{*}{ Location } & $\operatorname{Urban}(\mathrm{n}=32)$ & $4.6 \pm 2.6$ & Reference & \\
\hline & Suburban $(\mathrm{n}=3)$ & $5.0 \pm 2.6$ & $0.41(-2.75-3.56)$ & .80 \\
\hline & $\operatorname{Rural}(\mathrm{n}=1)$ & $0.0 \pm 0.0$ & $-4.59(-9.90-0.71)$ & .09 \\
\hline \multirow[t]{3}{*}{ Type of HTx center } & University teaching hospital $(n=30)$ & $4.6 \pm 2.8$ & Reference & \\
\hline & Regional/Community $(n=4)$ & $4.8 \pm 1.5$ & $0.18(-2.70-3.07)$ & 0.90 \\
\hline & Other $(\mathrm{n}=2)$ & $3.0 \pm 2.8$ & $-1.57(-5.52-2.39)$ & 0.43 \\
\hline
\end{tabular}

Obviously, de-implementation needs to be completed in a methodologically appropriate way with sufficient attention to stakeholder involvement and choice of appropriate de-implementation strategies. ${ }^{14}$ De-implementation could be monitored and evaluated by using standard cost data from local hospital management administrative systems combined with infection data from clinical information systems (ie, prevalence, incidence and type of infectious complications after HTx), and if available, also data from patient satisfaction and personnel surveys. Cost savings from de-implementation could be reinvested in adherence enhancing programs for effective standard precaution procedures as a cornerstone of post-operative management within transplantation and within the hospital overall. ${ }^{15}$

Limitations of this study are its cross-sectional design, the convenience sample of HTx centers, reliance on reporting of protective isolation practices by the HTx director only, and the lack of assessment of outcome data on infectious complications during the in-hospital phase in the participating programs. Future research should focus on a simultaneous assessment of protective isolation measures and clinical outcomes as this allows to evaluate effectiveness of protective isolation measures. Optimally, this type of study could use available outcome data of registries or cohort studies to assess the outcomes to reduce data collection burden.

Optimally investigators should have visited the $36 \mathrm{HTx}$ centers to investigate the current practice patterns in view of protective isolation measures; however, this was practically and financially not feasible. We trust that the standard methods we used in each center provide a solid basis to answer our research aims.

Our assessment included 12 protective isolation measures determined by previous publications as well as the expertise of the BRIGHT study team. The questionnaire assessed the prevalence of these protective isolation measures as yes/no, yet no additional information on specific aspects relevant for some of the protective isolation measures was collected (eg, specific procedures on changing linen, specifics of which high-touch surfaces were disinfected). As stated before, it was not feasible to visit the HTx centers and obtain a detailed view of all procedures. For future research, we suggest expanding the list of protective isolation measures, as some HTx centers use additional measures, such as shoe covers, beard covers, measures for food safety, or filters for water taps. Moreover, in addition to hand hygiene practices, other standard precautions, such as using aseptic techniques, should be included. Although important to ensure effective intervention control, we were unable to assess adherence to the protective isolation measures and standard precautions reported.

\section{CONCLUSIONS}

Protective isolation measures are still widely applied within heart transplant centers across the world, nevertheless there is limited current research providing evidence for the need of protective isolation measures. Future clinical guidelines for heart transplant management should include a statement of the need for strict adherence to standard infection prevention measures.

\section{References}

1. Ljungman P, Snydman D, Boeckh M, editors. Transplant infections, 4th ed. Basel (Switzerland): Springer; 2016. p. 101-12, 151-84, 823-43.

2. Kush KK, Cherikh WS, Chambers DC, Goldfarb S, Hayes D Jr., Kucheryavaya AY, et al. The International Thoracic Organ Transplant Registry of the International Society for Heart and Lung Transplantation: thirty-fifth adult heart transplantation report-2018; focus theme: multiorgan transplantation. J Heart Lung Transplant 2018;37:1155-68.

3. Mangini S, Alves BR, Silvestre OM, Pires PV, Pires LJ, Curiati MN, et al. Heart transplantation: review. Einstein 2015;13:310-8.

4. Centers for Disease Control and Prevention. Standard precautions for all patient care. Available from: https://www.cdc.gov/infectioncontrol/basics/standard-precautions.html. Accessed November 11, 2019.

5. Boaz MR, Bordignon S, Nesralla IA. The importance of preventive measures in the prophylaxis of infections in patients submitted to heart transplant during the first thirty postoperative days. Braz J Cardiovasc Surg 2006;21:188-93.

6. Costanzo MR, Dipchand A, Starling R, Anderson A, Chan M, Desai S, et al. International Society of Heart and Lung Transplantation. The ISHLT Guidelines for the care of heart transplant recipients. J Heart Lung Transplant 2010;29:914-56.

7. Walsh TR, Guttendorf J, Dummer S, Hardesty RL, Armitage JM, Kormos RL, et al. The value of protective isolation procedures in cardiac allograft recipients. Ann Thorac Surg 1989;47:539-45.

8. Gamberg P, Miller JL, Lough ME. Impact of protective isolation on the incidence of infection after heart transplantation. J Heart Transplant 1987;6:147-9.

9. Berben L, Denhaerynck K, Dobbels F, Engberg S, Vanhaecke J, Crespo-Leiro MG, et al. Building research initiative group: chronic illness management and adherence in transplantation (BRIGHT) study: study protocol. J Adv Nurs 2015;71:642-54.

10. Stehlik J, Edwards LB, Kucheryavaya AY, Aurora P, Christie JD, Kirk R, et al. The Registry of the International Society for Heart and Lung Transplantation: twenty-seventh official adult heart transplant report-2010. J Heart Lung Transplant 2010;29:1089-103.

11. Boraz RA, Myers R. A national survey of dental protocols for the patient with a cardiac transplant. Spec Care Dentist 1990;10:26-9.

12. Danzinger-Isakov LA, Faro A, Sweet S, Michaels MG, Aurora P, Mogayzel PJ Jr., et al. Variability in standard care for cytomegalovirus prevention and detection in pediatric lung transplantation: survey of eight pediatric lung transplant programs. Pediatr Transplant 2003; 7:469-73.

13. Ison MG, Stosor V. Transplantation of high-risk donor organs: a survey of US solid organ transplant center practices as reported by transplant infectious diseases physicians. Clin Transplant 2009;23:866-73.

14. Grol R, Wensing M, Eccles M, Davis D. Improving patient care: the implementation of change in health care. 2nd ed. Hoboken (NJ): John Wiley \& Sons; 2013.

15. De Geest S, Kesteloot K, Degryse I, Vanhaecke J. Hospital costs of protective isolation procedures in heart transplant recipients. J Heart Lung Transplant $1995 ; 14: 544-54$. 